This item was submitted to Loughborough's Research Repository by the author.

Items in Figshare are protected by copyright, with all rights reserved, unless otherwise indicated.

Control of multilevel voltage states in a hysteretic superconducting-quantuminterference-device ring-resonator system

PLEASE CITE THE PUBLISHED VERSION

http://dx.doi.org/10.1103/PhysRevE.72.056201

PUBLISHER

(C) American Physical Society

VERSION

VoR (Version of Record)

LICENCE

CC BY-NC-ND 4.0

REPOSITORY RECORD

Stiffell, P.B., Mark J. Everitt, T.D. Clark, and J.F. Ralph. 2019. "Control of Multilevel Voltage States in a Hysteretic Superconducting-quantum-interference-device Ring-resonator System”. figshare.

https://hdl.handle.net/2134/12063. 
This item was submitted to Loughborough's Institutional Repository (https://dspace.lboro.ac.uk/) by the author and is made available under the following Creative Commons Licence conditions.

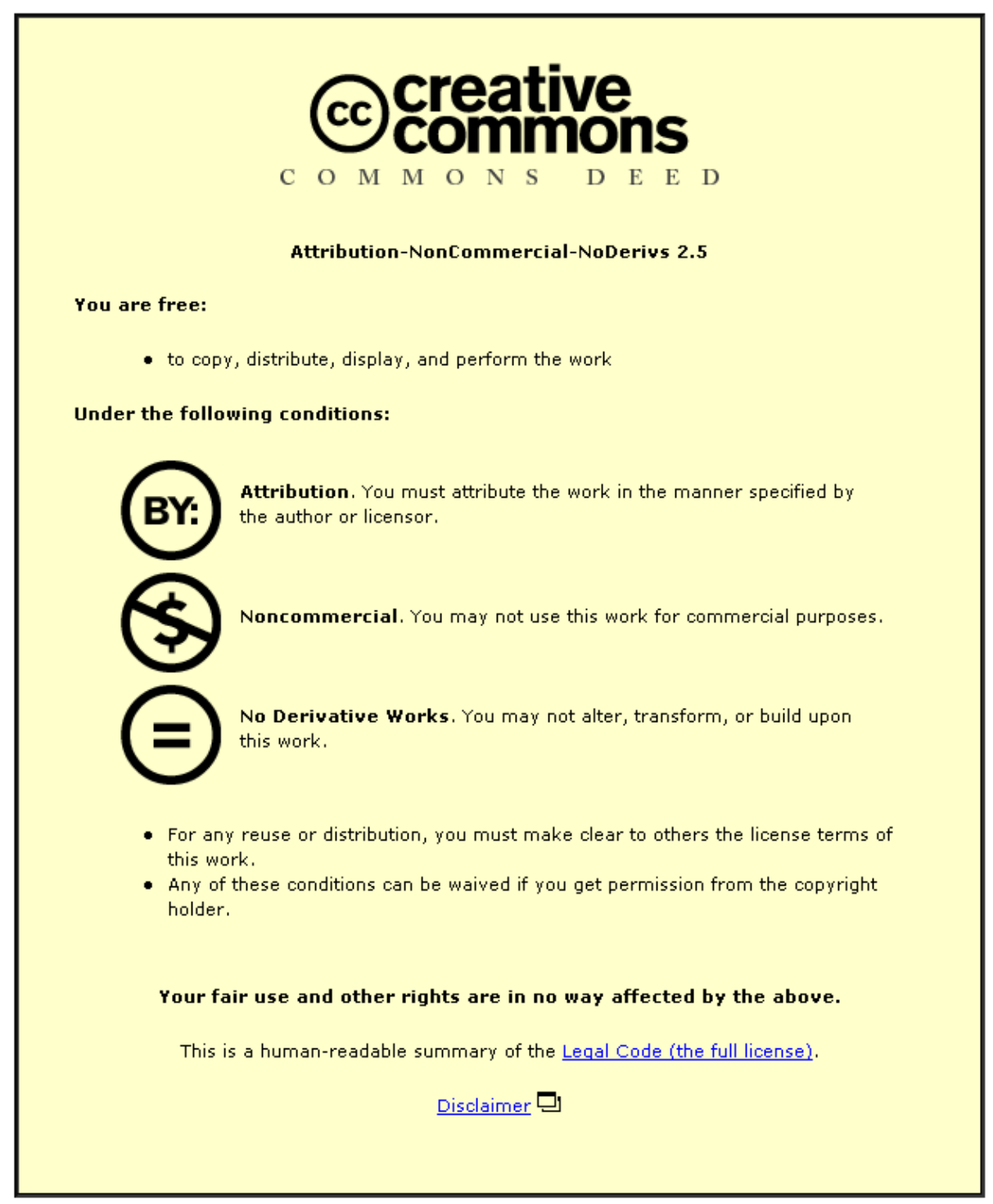

For the full text of this licence, please go to: http://creativecommons.org/licenses/by-nc-nd/2.5/ 


\title{
Control of multilevel voltage states in a hysteretic superconducting-quantum-interference-device ring-resonator system
}

\author{
P. Stiffell, ${ }^{1, *}$ M. J. Everitt, ${ }^{2,1, \dagger}$ T. D. Clark, ${ }^{1, \dagger}$ and J. F. Ralph ${ }^{3, \S}$ \\ ${ }^{1}$ School of Science and Technology, University of Sussex, Brighton, Sussex, BN1 9QT, United Kingdom \\ ${ }^{2}$ The British University in Egypt, 4 khaled Ibn Walid Street, Sheraton Helioplis Area, Cairo, Egypt \\ ${ }^{3}$ Department of Electrical Engineering and Electronics, University of Liverpool, Brownlow Hill, Liverpool, L69 3GJ, United Kingdom
}

(Received 17 May 2004; revised manuscript received 16 June 2005; published 1 November 2005)

\begin{abstract}
In this paper we study numerical solutions to the quasiclassical equations of motion for a superconductingquantum-interference device ring-radio frequency (rf) resonator system in the regime where the ring is highly hysteretic. In line with experiment, we show that for a suitable choice of ring circuit parameters the solutions to these equations of motion comprise sets of levels in the rf voltage-current dynamics of the coupled system. We further demonstrate that transitions, both up and down, between these levels can be controlled by voltage pulses applied to the system, thus opening up the possibility of high order (e.g., 10 state), multilevel logic and memory.
\end{abstract}

DOI: 10.1103/PhysRevE.72.056201

\section{INTRODUCTION}

In an earlier paper [1] we reported on phenomena generated by the nonlinear interaction of a superconductingquantum-interference device (SQUID) ring (here, a single Josephson weak link enclosed by a thick superconducting ring) with a parallel $L C$ resonant (tank) circuit. The block diagram for this coupled system is shown in Fig. 1. As has now become apparent, SQUID rings can display behavior ranging from fully quantum mechanical [2-5] through to quasiclassical [6-9] depending on the circuit parameters of the ring and the temperature $(T)$ of the environment. At temperatures of a few $\mathrm{K}$, and for relatively large weak link capacitances $\left(10^{-12}-10^{-13} \mathrm{~F}\right)$, it is well established $[6,8]$ that a SQUID ring (inductance $\Lambda$, weak link critical current $I_{c}$ ) can be treated quasiclassically, i.e., as a particle moving, with damping, in a cosine modulated parabolic potential,

$$
U\left(\Phi_{s}, \Phi_{x}\right)=\frac{\left(\Phi_{s}-\Phi_{x}\right)^{2}}{2 \Lambda}-\frac{I_{c} \Phi_{0}}{2 \pi} \cos \left(\frac{2 \pi \Phi_{s}}{\Phi_{0}}\right),
$$

where $\Phi_{x}$ is the external magnetic flux applied to the ring, $\Phi_{s}$ is the total included flux in the ring and $\Phi_{0}(\triangleq h / 2 e)$ is the superconducting flux quantum. In this quasiclassical regime it is usual practice to describe the SQUID ring, and its interactions, using the resistively shunted junction plus capacitance $(\mathrm{RSJ}+\mathrm{C})$ model $[8,10]$. Here, we denote the ring weak link effective capacitance by $C_{s}$ and the parallel link resistance by $R_{s}$. Furthermore, in making use of this model it is convenient to introduce the $\beta$-parameter for the SQUID ring $\left(\triangleq 2 \pi \Lambda I_{c} / \Phi_{0}\right)$. This allows us to distinguish between the parameter space in which $I_{c}$ is always a single-valued function of $\Phi_{x}$ (the inductive regime) and the hysteretic regime where $I_{c}$ can be multivalued in $\Phi_{x}[8,10]$.

\footnotetext{
*Electronic address: p.b.stiffell@physics.org

${ }^{\dagger}$ Electronic address: m.j.everitt@physics.org

"Electronic address: t.d.clark@sussex.ac.uk

${ }^{\S}$ Electronic address: jfralph@liverpool.ac.uk
}

PACS number(s): 05.45.-a

The cosine in the potential (1) leads to a superconducting screening current response in the SQUID ring which is manifestly a nonlinear, and $\Phi_{0}$-periodic, function of $\Phi_{s}$. When at least part of this external flux is time dependent, and applied through an inductively coupled resonant circuit, the ringresonator system can display nonlinear dynamical behavior which has been the focus of much research over the past three decades $[6,7,11]$. It is precisely this behavior that forms the basis of the well-known ac-biased SQUID magnetometer $[8,10,12]$. Although not invariably the case, it has been common practice [12] to make use of a radiofrequency ( $\mathrm{rf}$ $\approx 20 \mathrm{MHz}$ ), parallel $L C$ (tank circuit) resonator in these coupled magnetometer systems. In the work described here we adopt this frequency regime for the resonant circuit. Considered on its own, of course, the tank circuit is strictly linear, i.e., on resonance the $\mathrm{rf}$ voltage $\left(V_{\text {out }}\right)$ across it is linearly dependent on the level of rf drive input current $\left[I_{i n}(t)\right]$ applied. However, when a SQUID ring is coupled to such a tank circuit the situation can alter radically. With the SQUID ring potential above, and at finite critical current $I_{c}$, the screening supercurrent $I_{s}=\Phi_{s} / \Lambda$ flowing in the ring to oppose any externally applied flux $\Phi_{x}$ is a nonlinear function of this flux. Since both the SQUID ring and the tank circuit are macroscopic in nature, the back reaction through the inductive coupling leads to the system displaying nonlinear, even chaotic, dynamics. In general the stronger the nonlinearity in $I_{s}\left(\Phi_{x}\right)$, the more pronounced the nonlinear behavior can be. This nonlinear behavior is easily seen in plots of $V_{\text {out }}$ versus $I_{i n}(t)$, these also being dependent on the level of static or quasistatic bias flux $\left(\Phi_{x}\right)$ applied to the SQUID ring. In

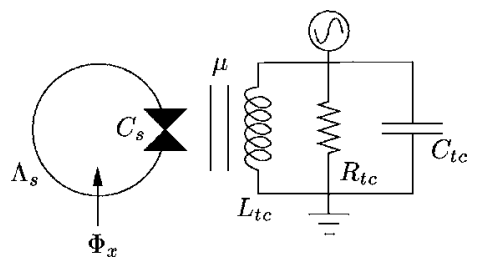

FIG. 1. Block diagram for an inductively coupled SQUID ringtank circuit system. 

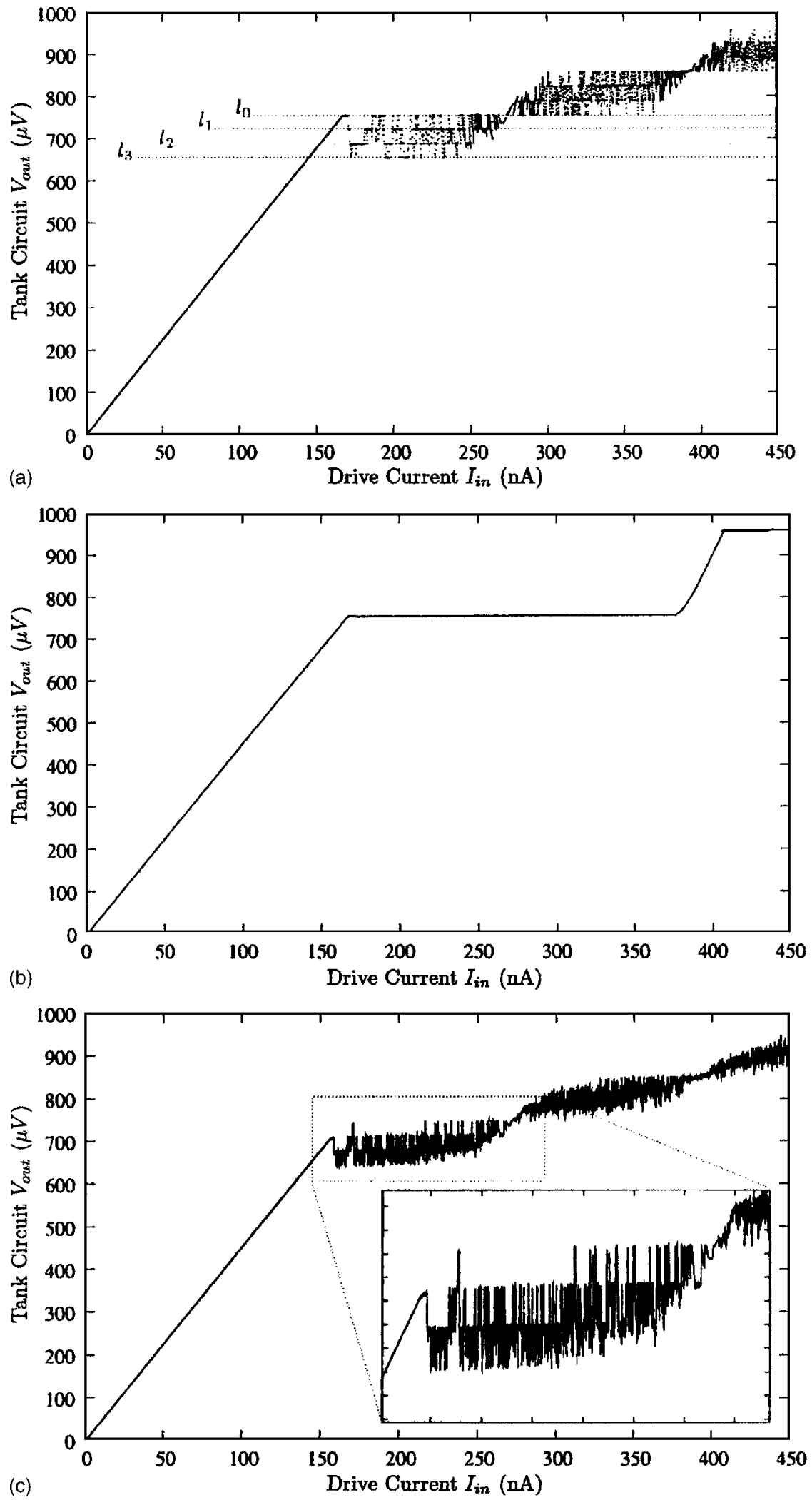

FIG. 2. Dynamical rf voltage $\left(V_{\text {out }}\right)$ versus rf current $\left(I_{\text {in }}\right)$ characteristics for a highly hysteretic $(\beta=137)$ SQUID ring-tank circuit system for (a) relatively low integration accuracy with $\alpha \approx 0.16 \mathrm{pA}, \beta \approx 3.4 \mathrm{pA}$ (both corresponding to $4.2 \mathrm{~K}$ ) and $\gamma=0.0 \mathrm{~A}$, (b) as for (a) but with high integration accuracy, and (c) a high integration accuracy calculation with $\gamma \approx 7 \mathrm{nA}$ and $\alpha=\beta=0.0 \mathrm{~A}(0.0 \mathrm{~K})$. All three computations were performed with the following circuit parameters for the ring: $C_{s}=1 \times 10^{-13} \mathrm{~F}, \Lambda_{s}=6 \times 10^{-10} \mathrm{H}, R_{s}=10 \Omega, I_{c}=75.2 \mu \mathrm{A}$, and $\Phi_{x}$ $=0.0 \Phi_{0}$ (modulo $n \Phi_{0}, n$ integer); and the tank circuit: $C_{t c}=7.6 \times 10^{-10} \mathrm{~F}, L_{t c}=63 \mathrm{nH}$ and $\mu\left(=M^{2} / L_{t c} \Lambda\right)=0.0087$, a bare tank circuit resonant frequency $23 \mathrm{MHz}$. 
SQUID magnetometer systems these characteristics display essentially constant $\mathrm{rf}$ voltage steps in the (time averaged) $V_{\text {out }}$ versus $I_{\text {in }}(t)$ at intervals periodic in $I_{i n}$. These SQUID ring voltage steps are modulated in a $\Phi_{0}$-periodic manner by the external bias flux $[8,12,13]$. The ring-tank circuit system is described dynamically by two (coupled) equations of motion, one for the tank circuit and the other for the ring. These are given by

$$
\begin{gathered}
C_{t c} \frac{d^{2} \Phi_{t c}}{d t^{2}}+\frac{1}{R_{t c}} \frac{d \Phi_{t c}}{d t}+\frac{\Phi_{t c}}{L_{t c}\left(1-K^{2}\right)} \\
=I_{i n}(t)+\frac{\mu \Phi_{s}}{\Lambda\left(1-K^{2}\right)} \quad \text { (tank circuit), } \\
C_{s} \frac{d^{2} \Phi_{s}}{d t^{2}}+\frac{1}{R_{s}} \frac{d \Phi_{s}}{d t}+I_{c} \sin \left(\frac{2 \pi \Phi_{s}}{\Phi_{0}}\right)+\frac{\Phi_{s}}{\Lambda\left(1-K^{2}\right)} \\
=\frac{\mu \Phi_{t c}}{\Lambda\left(1-K^{2}\right)} \quad \text { (SQUID ring), }
\end{gathered}
$$

where the subscripts $t c$ and $s$ refer, respectively, to the tank circuit and the SQUID ring and $\mu\left(=M / L_{t c}\right)$ is the fraction of the flux coupled between the ring and the tank circuit. Thus, $C_{t c}$ and $L_{t c}$ are, respectively, the tank circuit capacitance and inductance, $\Phi_{t c}$ is the flux in the tank circuit inductor, $R_{t c}$ is the resistance of the parallel tank circuit on resonance and $K\left(=\sqrt{M^{2} / \Lambda L_{t c}}\right)$ quantifies the strength of the inductive coupling between the ring and tank circuit with a mutual inductance of $M$. In (2) and (3) the last terms on the right-hand sides of these equations describe the back reaction between the ring and the tank circuit.

In the original paper [1] we used (2) and (3) to compute the $V_{\text {out }}$ versus $I_{\text {in }}$ dynamics of the ring-tank circuit system in the highly hysteretic, strongly underdamped, regime to model the observed experimental behavior. What was recorded experimentally was a succession of plateau regions in the time averaged $V_{\text {out }}$ versus $I_{\text {in }}$ characteristics of the ringtank circuit system. Each plateau region consisted of a set of parallel steps at regular separations along the $I_{i n}$ axis. The lengths of these steps along this axis were much greater than those observed in standard hysteretic SQUID magnetometer characteristics, where, typically, $\beta \approx$ a few $[8,12]$. In addition, for each individual plateau the rf voltage $\left(V_{\text {out }}\right)$ at which these steps occurred varied $\Phi_{0}$ periodically in $\Phi_{s}$. In experiment [1] the ring-tank circuit system was observed to jump stochastically [14-16] between the various steps associated with each plateau. Since, in practice, it was easy experimentally to access circuit parameters in which many steps per plateau region could be seen (a maximum of 19 steps/plateau was recorded over the course of these experiments), there seemed a possibility that the plateaux and steps could be utilized to create multilevel logic as an alternative to the standard binary logic. We found that with simulated low temperature noise on the tank circuit drive current, introduced via a noise distribution for a thermal bath, the solutions to these coupled equations of motion modeled our experimental results very well, including the stochastic jumping between steps on particular plateaux. We argued in the paper [1] that when the SQUID ring is sufficiently underdamped it no longer follows the potential (1) adiabatically as the rf flux coupled in from the tank circuit changes with time. Essentially, it is this nonadiabatic response that generates the large multistep plateaux in the ring-tank circuit $V_{\text {out }}$ versus $I_{\text {in }}$ characteristics, each step corresponding to a different flux jump trajectory (local well to local well) in (1). Nevertheless, even though we had demonstrated that the underdamped RSJ $+\mathrm{C}$ description could model the experimentally observed plateaux and steps, the stochastic jumping between steps generated in the theoretical calculations required more explanation. In the experiments it was assumed that these jumping processes were caused by ambient thermal current noise in the SQUID ring. However, for technical reasons we were not able to measure the full spectral density function of this noise and with the computer power then available to us we could not be certain that the stochastic jumping processes seen in the simulations did not arise because of computational inaccuracies. Different calculations, at much higher accuracy, are the basis of the work reported here. At this level we have been able to show that, from the viewpoint of the simulations presented in our previous paper [1], the jumping processes, as modeled in that work, arose due to the build up of computational error in the numerical integration for the evolution of the system. Repeating the calculations with the same level of thermal noise (with appropriate care taken to compensate for the change in accuracy, and concomitant reduction in stepsize, used), and eliminating this potential problem by using more accurate integration methods, only single steps are generated in each plateau region. Nevertheless, it was clear from experiment that the ring-tank circuit system could be perturbed to generate jumps. We now know that our original assumption, that these voltage levels were due to the thermal noise applied to that SQUID ring, was incorrect. However, we have identified a suitable noise source within the external ring bias flux $\Phi_{x}$ that, independent of our integration accuracy, reproduces very well the voltage dynamics of our experimental system. This independence was tested by increasing the integration accuracy used by several orders of magnitude resulting in considerably smaller stepsizes. We present these results in the next section before proceeding to consider in some detail the underlying SQUID ring dynamics, which cause this behavior. Finally we show that in a low noise environment we can control the voltage state of the tank circuit through the SQUID ring in a way that points to possible device applications in multilevel logic.

\section{SQUID RING-TANK CIRCUIT DYNAMICS IN THE HIGHLY HYSTERETIC REGIME}

\section{A. Introduction}

In this work we have modeled the dynamics of the ringtank circuit system in the presence of noise by computing realizations of the Langevin equation though a four dimensional stochastic (itô) differential equation. The form of this equation is determined by Eqs. (2) and (3) and is given by [17]

$$
d \boldsymbol{\varphi}(t)=\mathbf{A} \boldsymbol{\varphi}(t) d t+\mathbf{f}(t) d t+\mathbf{n}_{T} d B_{t}^{T}+\mathbf{n}_{\Phi} d B_{t}^{\Phi},
$$

where 


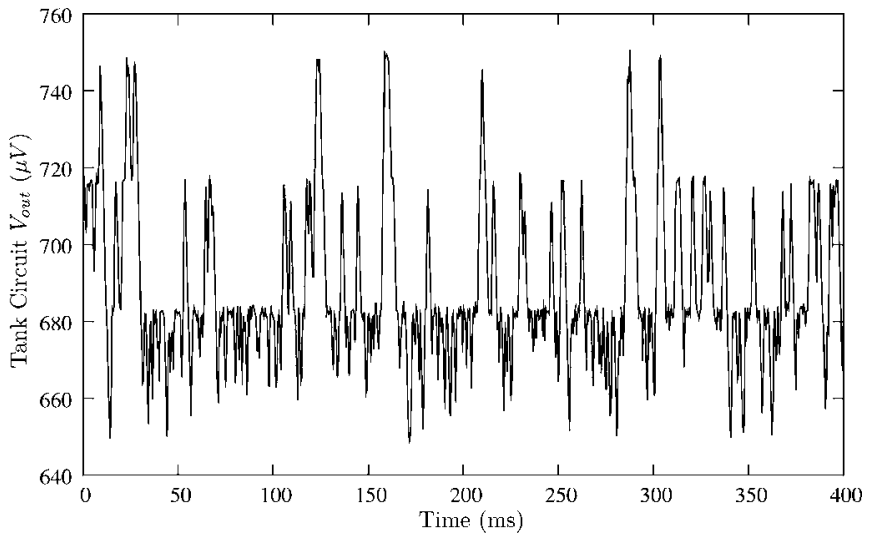

FIG. 3. Dynamical rf voltage $\left(V_{\text {out }}\right)$ versus time for the SQUID ring ring-tank circuit system of Fig. 2(c) at a drive current of $230 \mathrm{nA}$.

$$
\begin{gathered}
d \boldsymbol{\varphi}(t)=\left(\begin{array}{c}
d \Phi_{t} \\
d \dot{\Phi}_{t} \\
d \Phi_{s} \\
d \dot{\Phi}_{s}
\end{array}\right), \quad \boldsymbol{\varphi}(t)=\left(\begin{array}{c}
\Phi_{t} \\
\dot{\Phi}_{t} \\
\Phi_{s} \\
\dot{\Phi}_{s}
\end{array}\right), \\
\mathbf{A}=\left(\begin{array}{cccc}
0 & 1 & 0 & 0 \\
\frac{-1}{L_{t}^{*} C_{t}} & \frac{-1}{R_{t} C_{t}} & \frac{\mu}{L_{s}^{*} C_{t}} & 0 \\
0 & 0 & 0 & 1 \\
\frac{\mu}{L_{s}^{*} C_{s}} & 0 & \frac{-1}{L_{s}^{*} C_{s}} & \frac{-1}{R_{s} C_{s}}
\end{array}\right)
\end{gathered}
$$

[here $L_{s}^{*}=\Lambda\left(1-K^{2}\right)$ and $L_{t}^{*}=L_{t}\left(1-K^{2}\right)$,

$$
\begin{aligned}
\mathbf{f}(t) & =\left(\begin{array}{c}
0 \\
I_{\text {in }}(t) / C_{t} \\
0 \\
-\left(I_{c} / C_{s}\right) \sin \left(2 \pi \Phi_{s} / \Phi_{0}\right)
\end{array}\right), \\
\mathbf{n}_{T} & =\left(\begin{array}{c}
0 \\
\alpha / C_{t} \\
0 \\
\beta / C_{s}
\end{array}\right), \quad \mathbf{n}_{\Phi}=\left(\begin{array}{c}
0 \\
0 \\
0 \\
\gamma / C_{s}
\end{array}\right)
\end{aligned}
$$

and $B_{t}^{T}$ and $B_{t}^{\Phi}$ are one-dimensional Brownian motions of zero mean with variances defined by $\alpha=\sqrt{2 k_{B} T / R_{t}}, \beta$ $=\sqrt{2 k_{B} T / R_{s}}$ (i.e., the level of thermal noise on the system [18]) and $\gamma=\Phi_{\text {noise }} / L_{s}^{*}$ (i.e., modeling the noise on the bias flux $\Phi_{x}$ ).

We have chosen suitable ring and tank circuit parameters for this semiclassical regime, specifically for the SQUID ring: $\Lambda_{s}=6 \times 10^{-10} \mathrm{H}, I_{c}=75.2 \mu \mathrm{A}(\beta=137), C_{s}=10^{-13} \mathrm{~F}$, $R_{s}=10 \Omega$ and for the tank circuit: $C_{t c}=7.6 \times 10^{-10} \mathrm{~F}, L_{t c}$ $=63 \mathrm{nH}$ and a quality factor $Q$ of 500, the latter parameters yielding a bare (uncoupled to the ring) tank circuit resonant frequency of $23 \mathrm{MHz}$. We also set $\mu\left(=M^{2} / L_{t c} \Lambda\right.$; $)=0.0087$, a coupling quite typical of low noise SQUID ring-tank circuit systems. These are the circuit parameters used throughout this paper.

As stated above, with access to more computational power we considered it important to establish the origin of the stochastic jumping in the computer simulations as previously published [1]. As in our previous work, we compute the $V_{\text {out }}$ versus $I_{\text {in }}$ characteristics for a SQUID ring-tank circuit system. In Fig. 2 we show three sets of computed $V_{\text {out }}$ versus $I_{i n}$ characteristics using our chosen ring and tank circuit parameters. With these values of $R_{s}$ and $C_{s}$ the SQUID is underdamped. The originally reported characteristic [1], calculated at relatively low accuracy using a fourth order Runge-Kutta numerical integration routine with an adaptive step size algorithm, is plotted in Fig. 2(a) for the first plateau region. In generating this characteristic, $4.2 \mathrm{~K}$ current noise $(\alpha \approx 0.16 \mathrm{pA}, \beta \approx 3.4 \mathrm{pA}$, and $\gamma=0.0 \mathrm{~A})$, has been introduced and the bias flux has been set at $\Phi_{x}=0.0 \Phi_{0}$ (modulo $\left.n \Phi_{0}\right)$. Here, the multistep solutions on the first plateau, and the stochastic jumping between these, are perfectly clear. Since the initial calculations were performed, we have had access to much greater computational power and this has allowed us to apply significantly higher accuracy to our numerical integration. Subsequently we have found that this greatly improved accuracy leads to the suppression of the jumps between the multiple levels in the $V_{\text {out }}$ versus $I_{\text {in }}$ characteristics. This is very apparent in the next solution shown in Fig. 2(b) for which we used exactly the same SQUID and tank circuit parameters as in the characteristic of Fig. 2(a) and simply increased the accuracy of our integration routine. The obviously apparent differences between Figs. 2(a) and 2(b) clearly shows that it was inaccuracy in our integration, and not the thermal current noise, that has led to the levels of behavior found in Fig. 2(a). A further, detailed, investigation was then performed from which we have determined that the most likely source of noise that would generate the voltagecurrent characteristics observed experimentally is that of a noise contribution to the dc bias flux $\Phi_{x}$. We show in Fig. 2(c), for a noise level of $\Phi_{\text {noise }}=0.006 \Phi_{0}$, corresponding to a current of $\gamma \approx 21 \mathrm{nA}$ in the ring (and $\alpha=\beta=0.0 \mathrm{~A}$ ) a voltagecurrent characteristic that matches very well with those originally reported in [1]. We note that the level structure seen in Fig. 2(c) is less pronounced than that seen in Fig. 2(a). This is explained by the fact that the form of Fig. 2(a) is a byproduct of inaccurate integration while the the level structure in Fig. 2(c) is found from an actual realization of Eq. (4). Furthermore the results of Fig. 2(c) are in far better agreement with previous experimental results than those of Fig. 2(a). Additionally, in Fig. 3, we display the evolution of the rf voltage as a function of time; again these results agree very well with the experimental data presented in [1]. Furthermore, we have determined that below a noise amplitude of $\Phi_{\text {noise }}=0.002 \Phi_{0}$, i.e., $\gamma \approx 7 \mathrm{nA}$, these voltage jumps are completely suppressed. In order to understand how it is possible to access these discrete voltage levels, as we shall indeed demonstrate later, we will now proceed to consider the actual mechanism by which the tank circuit can realize these various levels.

\section{B. Underlying dynamics}

In considering the dynamical basis for the plateaux (and the step levels) it is helpful to examine the way in which the 


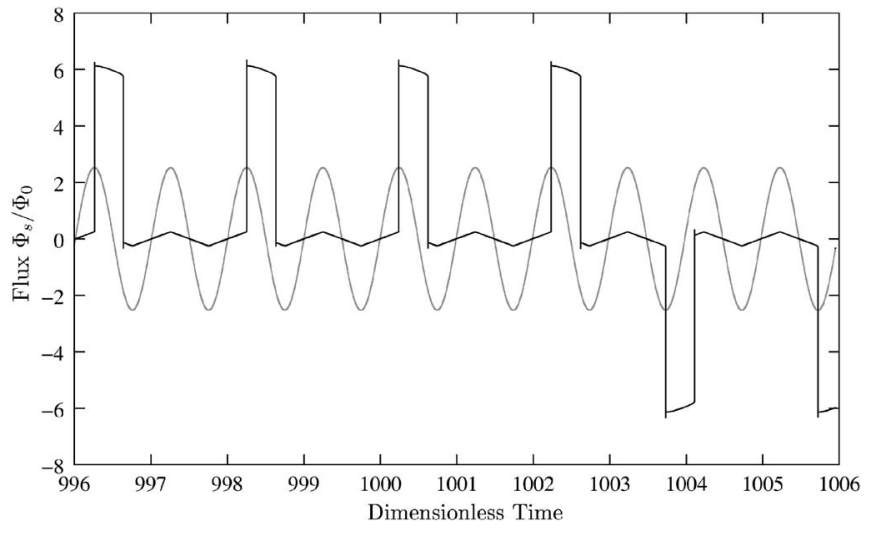

FIG. 4. Time dependence of the normalized SQUID ring flux $\Phi_{s} / \Phi_{0}$ as a function of time, normalized to the tank circuit period $2 \pi \sqrt{L_{t c} C_{t c}}$.

SQUID ring flux $\left(\Phi_{s}\right)$ changes in time with the rf drive current $\left(I_{i n}\right)$. Such a response is shown in Fig. 4 for a maximum (sinusoidal) drive flux in the tank circuit of $13.5 \Phi_{0}$ peak to peak (equivalent to a drive flux in the SQUID ring of $\left.0.1175 \Phi_{0}\right)$. In this figure the SQUID ring flux is normalized to $\Phi_{0}$ and the time axis is plotted in units of the reciprocal tank circuit period $\left(2 \pi \sqrt{L_{t c} C_{t c}}\right)$ and, for comparative timing purposes, we show the form of $I_{\text {in }}$ in light grey. What this computed plot demonstrates is that, for the most part, the oscillating flux $\left(\Phi_{s}\right)$ in the underdamped SQUID ring corresponds to the ring being confined to a single well. In Fig. 4 this single well SQUID ring flux response is shown oscillating over small amplitudes around $\Phi_{s} / \Phi_{0}=0$. However, when the drive current reaches maximum amplitude it is found that the SQUID ring can move outside the confines of a single well and make more extended trajectories in the potential (1), i.e., the flux in the ring can jump (nonadiabatically) several $\Phi_{0}$ in $\Phi_{s}$ space between initial and distant target wells [19]. We now provide some illustrative examples of stable solutions of Eqs. (2) and (3) which give rise to the different voltage levels seen in Fig. 2(a).

In the particular example of Fig. 4 these jumps take place over \pm six wells in $\Phi_{s}$ space relative to the lowest energy well in the potential. Transposed to the rf dynamics of the coupled system, each multi- $\Phi_{0}$ traversal leads to one of the set of plateau step levels in $V_{\text {out }}$ versus $I_{i n}$, as exemplified in Fig. 2. Examples of these multi- $\Phi_{0}$ trajectories (jumps) in the SQUID ring potential can be seen in Fig. 5, again for the system of Fig. 2. Taking the origin as the lowest well $\left(w_{0}\right)$ in this potential, the SQUID flux trajectories shown in Figs. 4 and 5 correspond to the level denoted $l_{0}$ in Fig. 2(a). If the origin is displaced to the next lowest well $\left(w_{ \pm 1}\right)$ in the potential, the $V_{\text {out }}$ versus $I_{\text {in }}$ characteristic of the coupled system moves down by one level from the original solution. This level is denoted $l_{1}$ in the solutions plotted in Fig. 2(a). Moving the origin again to the next lowest well $\left(w_{ \pm 2}\right)$ repeats the process, i.e., the dynamics now correspond to the level denoted by $l_{2}$ in the solutions plotted in Fig. 2(a). Again, moving the origin to the next lowest well $\left(w_{ \pm 3}\right)$ generates the level denoted $l_{3}$ in Fig. 2(a). However, in our example of a $6 \Phi_{0}$ excursion this level $\left(l_{3}\right)$ is equivalent to having displaced our origin to the middle well. We find that beyond this central well in the excursion, attempting to localize our trajectory around one of the remaining three wells simply re-

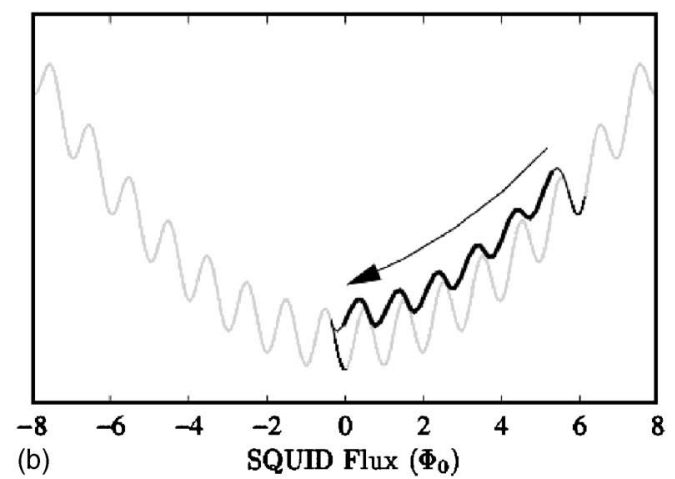

(b)

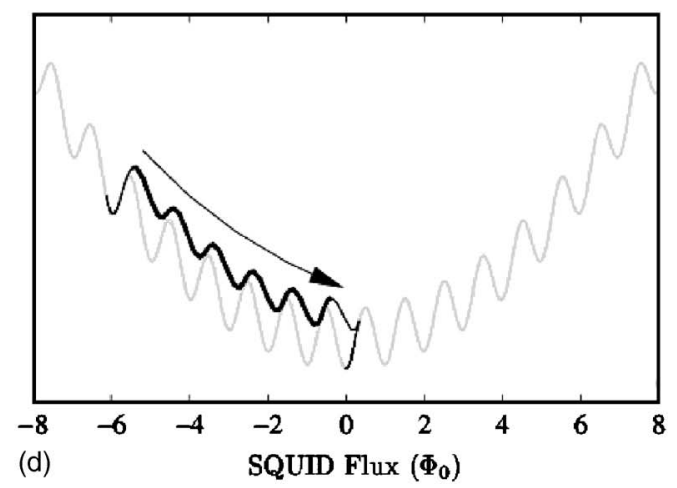

FIG. 5. The SQUID ring potential for the system is shown in grey for (a) a positive $\Phi_{s}$ going, outward, multi- $\Phi_{0}$ trajectory; (b) return trajectory for (a); (c) a negative going, multi- $\Phi_{0}$ trajectory; (d) return trajectory for (c). 
verses the shift between the levels on the plateau in Fig. 2(a), i.e., starting at $l_{3}$ and returning to $l_{0}$ through levels $l_{2}$ and $l_{1}$. To illuminate this discussion, we can examine, for level $l_{0}$, the excursions in the potential shown in Fig. 5. Starting in the absolute minimum well of the potential at $\Phi_{s} / \Phi_{0}=0$, with $\Phi_{x}=0$ (modulo $n \Phi_{0}$ ), the ring can either execute local motion in this well or occasionally move several $\Phi_{0}$ to a target well. With the ring potential plotted in light grey in Fig. 5, we show by computation in Fig. 5(a) one such extended trajectory, starting in the lowest well and followed by a positive going trajectory in $\Phi_{s}$. In this example, having reached the target well the ring completes almost half a tank circuit period in this well before returning to its original starting point in $\Phi_{s}$, as shown in Fig. 5(b). This mirrors the dynamical behavior shown in the first four jumps of Fig. 4. It is also important to note that these excursions do not occur every time the rf drive flux reaches its maximum amplitude, although as the amplitude of the $\mathrm{rf}$ drive grows this process happens more frequently. Further multi- $\Phi_{0}$ excursions in the ring potential, but now for negative going $\Phi_{s}$, can be seen in Figs. 5(c) and 5(d) for the outward and return paths, respectively. These correspond to the last two jumps in Fig. 4. For reasons of clarity we have taken a snapshot of the dynamics of the SQUID ring over a short interval in time. However, although it is not possible to observe this from Fig. 4, this is a segment of one of several stable, periodic, solutions to Eq. (4) in the absence of noise. We note that in general the target well in the SQUID ring is not symmetric about its center (minimum) and this is seen to affect the lifetime of the ring in its target well, as evidenced in plots presented in Fig. 4. In essence the consequence of this is that the ring can reach a new traversal (target) point in drive flux before it has completed half a $\Phi_{0}$ period in $\Phi_{s}$. This phenomenon, arising from the localization in a nonsymmetric well in the SQUID ring potential, means that the excursions in $\Phi_{s}$ are only made in one direction.

\section{Multilevel logic}

With the details of Fig. 5 in mind, and as a possible means to navigate the solutions of (2) and (3), we now consider the effect of rapidly changing voltages applied to the SQUID ring. In order make this relatively simple, and physically transparent, we simulate the application of appropriately shaped voltage pulses to the SQUID ring, and follow the tank circuit voltage $\left(V_{\text {out }}\right)$ response. In our example we shall now consider positive amplitude trapezoidal voltage pulses (inset in Fig. 6) with an upper voltage state time duration of 0.01 tank circuit periods which is activated during the flux traversal shown in bold in Fig. 5(d). We found that it was only within such highlighted activation regions as illustrated in Figs. 5(a)-5(d) that the system could be made to respond to these voltage pulses, leading to a level change in $V_{\text {out }}$. For reference we term pulses with positive amplitude as $A$ type pulse and, conversely, negative amplitude pulses are denoted as $B$ type. In Fig. 6 we have plotted the computed tank circuit voltage response as a function of normalized tank circuit time (i.e., in terms of the tank circuit period) for the rf drive amplitude $\left(I_{i n}=222 \mathrm{nA}\right)$ set in the middle of the first plateau

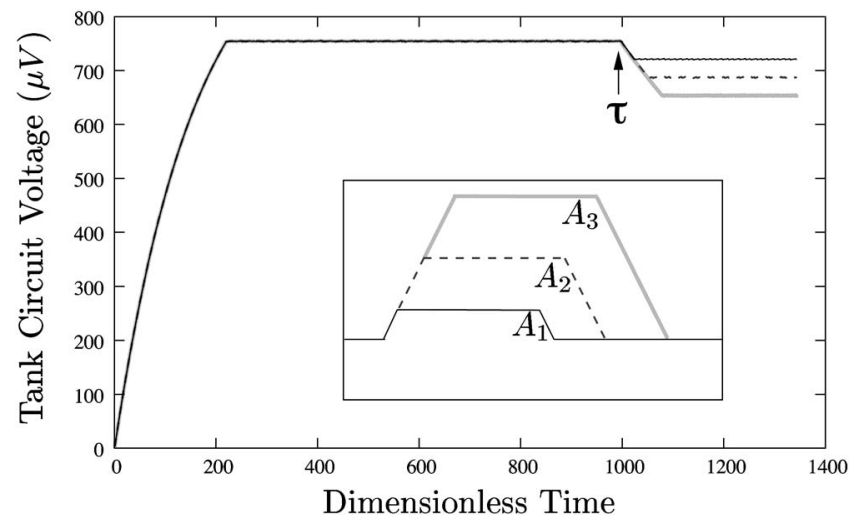

FIG. 6. $V_{\text {out }}$ versus normalized time characteristics for the SQUID ring-tank circuit system of Fig. 2 showing the controlled step to step jumping which can be induced using suitably configured positive (A-type) ramp pulses in the absence of extraneous voltage noise; here we show the effect of increasing the ramp amplitude to induce jumps over one, two or three step intervals.

for the level $l_{0}$ as denoted in Fig. 2(a). In this calculation there was no extraneous flux or voltage noise present and the system was allowed to reach a steady state within this plateau (we note that the initial sharp rise at the beginning of the voltage dynamics is due to transient behavior). However, after a set time, denoted by $\tau$ in Fig. 6, a voltage pulse of the form shown inset in this figure was applied. Here, the amplitudes of the pulses $A_{i}, i=1,2,3$ used were 15,75 , and $120 \mu \mathrm{V}$, respectively. As is demonstrated in Fig. 6, the application of a single voltage pulse can change the voltage level of our system by one or more levels at a time, as summarized in Table I. If the noiseless (or, in practice, a low enough voltage noise) situation can be realized experimentally, the computational results of Fig. 6 indicate that multilevel logic, based on SQUID ring-resonator systems could be a feasible proposition. If so, it is reasonable to assume that other forms of voltage pulse could be used for this purpose. With regard to our example, we note that the number of levels on the plateaux depends on the ring parameter values. In the example of Fig. 6 this is four but could be significantly larger. To further illustrate the control possible we show in Fig. 7 the result of applying two $A$-type pulses, in time sequence, to the SQUID ring-tank circuit system.

Of course, the activation pulse in voltage can be negative (B-type) as well as positive. The effect of using such a pulse after an A-type pulse is shown in Fig. 8 with the form of the $B$-type pulse shown explicitly in the inset of this figure. This shows that in the absence of extraneous noise, and by a suitable choice of voltage pulse form, we can move at will be-

TABLE I. Summary of pulse amplitude ranges $(\mu \mathrm{V})$ as described in the text.

\begin{tabular}{cccc}
\hline \hline Pulse type & Lower bound & Upper bound & Value used \\
\hline$A_{1}$ & 15 & 55 & 15 \\
$A_{2}$ & 56 & 90 & 75 \\
$A_{3}$ & 91 & 126 & 120 \\
\hline \hline
\end{tabular}




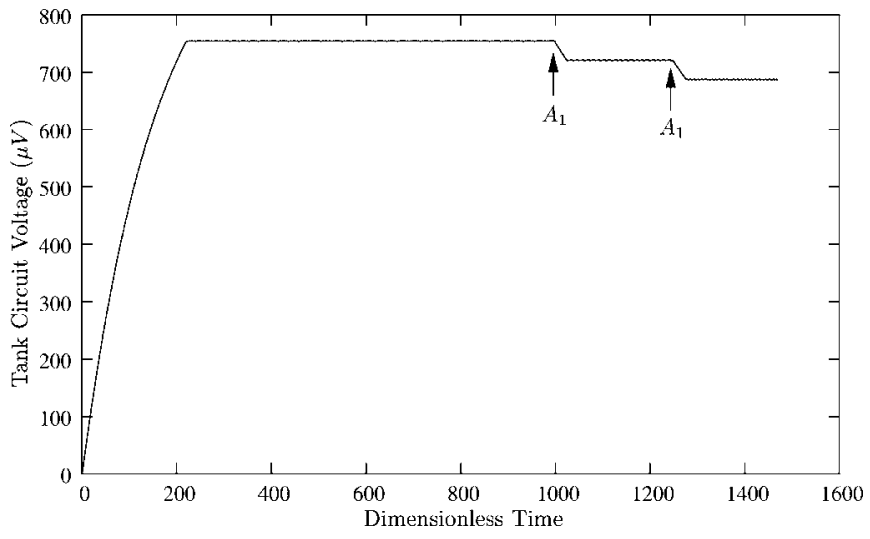

FIG. 7. $V_{\text {out }}$ versus normalized time characteristics for the SQUID ring-tank circuit system of Fig. 2 showing the effect of two sequential $A$-type voltage pulses in the absence of extraneous voltage noise; as is apparent these step jumps can be induced in a controlled manner.

tween the steps on any particular plateau in $V_{\text {out }}$ versus $I_{\text {in }}$. To demonstrate that we can induce step to step jumping at essentially any time of our choosing, we show in Fig. 9 a sequence of jumps induced by a sequence of $A$-type pulses spaced by different time intervals. We note that the application of the last pulse causes the tank circuit voltage to step up rather than down.

The computed solutions of Figs. 6-9 show very well the level to level jumping induced by various pulse sequences applied to a highly hysteretic (large $\beta$ ) SQUID ring-tank circuit system. These solutions, which are the end result of very non-linear interactions between the ring and the tank circuit, may be appreciated more clearly by the following qualitative argument. As regards the jumping process between levels on any particular plateau in $V_{\text {out }}$ versus $I_{\text {in }}$, the system operates cyclically from an initial (local) well in the SQUID potential (for example, the $w_{0}$ well in the potential of Fig. 5). This is shown diagrammatically in Fig. 10. The way in which movement between wells in the SQUID potential relates to a given voltage level within a particular plateau can be determined by thinking of each well being mapped onto

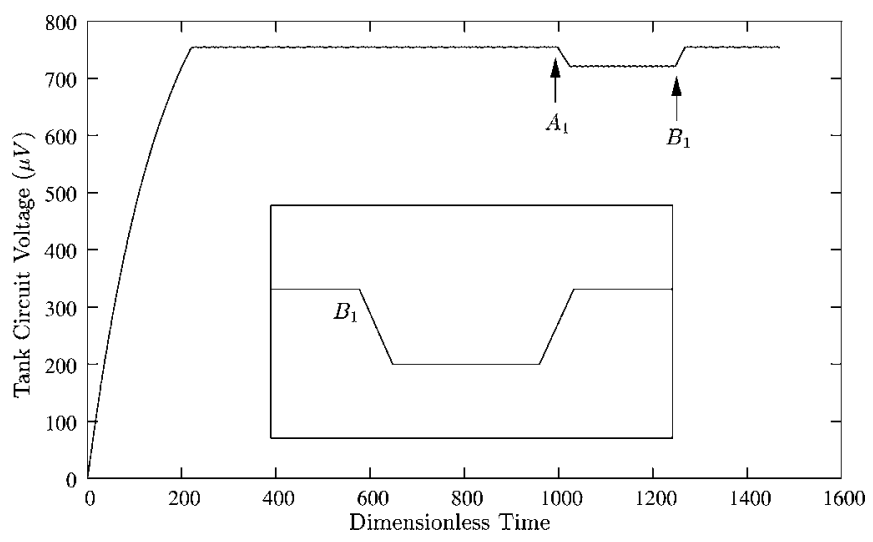

FIG. 8. $V_{\text {out }}$ versus normalized time characteristics for the SQUID ring-tank circuit system of Fig. 2 in the absence of extraneous voltage noise showing the effect of applying sequential $A$ and $B$-type voltage ramps.

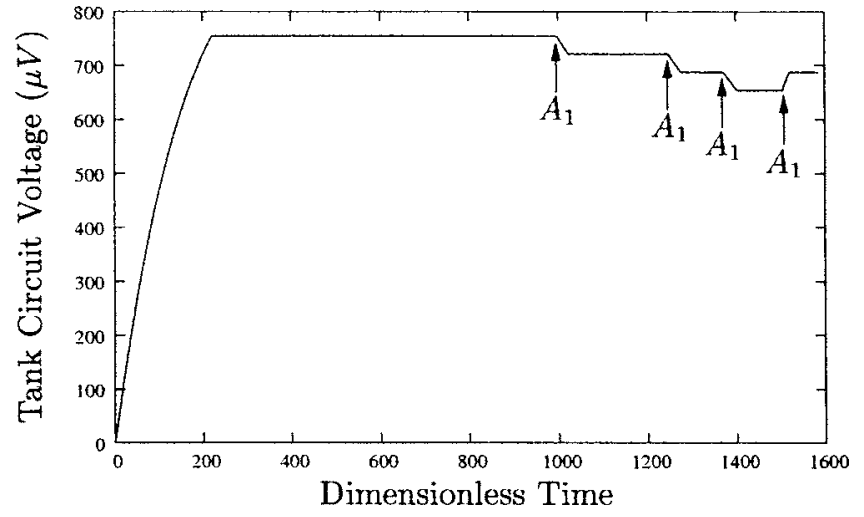

FIG. 9. $V_{\text {out }}$ versus normalized time characteristics for the SQUID ring-tank circuit system of Fig. 2 in the absence of extraneous voltage noise showing the effect of applying sequentially at differing time intervals as a set $A$-type voltage ramps.

one of the vertices of a polygon (where the order of the polygon is dependent on the parameter values of the ringtank circuit system under investigation). This is illustrated in Fig. 10(a) for the system considered throughout this paper with the vertices labeled $v_{i}$ for $i=0, \ldots, 5$ and where the mapping is applied cyclically from the lowest (reference) well of the potential onto each vertex (for our example using the labels for the wells $w_{i}$ in Fig. 5(a) we have the mapping $w_{i} \rightarrow v_{\text {imod6 }}$ for $i \in \mathbb{Z}$ ). Here, we can find the particular tank circuit voltage level associated with each vertex by projection onto the horizontal axis. For example, the vertices $v_{1}$ and $v_{5}$ correspond to having the SQUID ring-tank circuit system in level $l_{1}$ in Fig. 2. Clearly, therefore, if the system is moved through an appropriate number of wells it will return to the same state of operation in which it began.

Using this graphical map as the guide, in this paper we have investigated a system with 4 levels, corresponding to Fig. 10(a). We note that in this example the system must traverse 6 wells in the potential before it returns to its original state of operation. If we choose different SQUID parameters the system can access a variable number of wells within

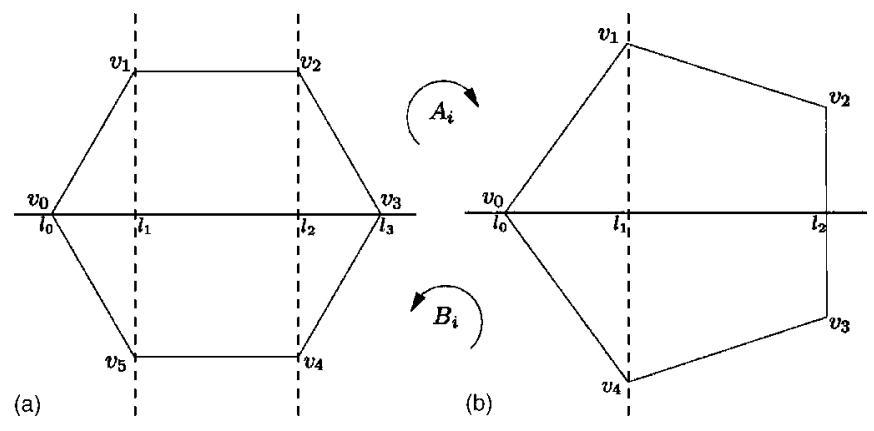

FIG. 10. Graphical representation in the manner in which the wells in the SQUID potential correspond to voltage levels in the $V_{\text {out }}$ versus $I_{\text {in }}$ characteristic of a highly hysteretic SQUID ring-tank circuit system. The vertices around the polygons indicate which well (in a six well example-see Fig. 5) the SQUID oscillation is localized in, while the letters on the projection denote which one of the set of levels on a particular plateau in $V_{\text {out }}$ versus $I_{\text {in }}$ the coupled system occupies. See text for more details. 
its cycle of operation. However, the system will always display a symmetry about the horizontal axis. For comparison, we show in Fig. 10(b) a diagram illustrating the situation where the ring-tank circuit system traverses an odd number of wells in the SQUID potential, in this case five.

By the application of suitable voltage pulses the system can be moved around the vertices of the polygon, so translating the SQUID ring from one potential well to another. Thus, in Fig. 10(a) application of positive (A-type) pulses causes the ring to move around the polygon in a clockwise direction, while negative ( $B$-type) pulses lead to anticlockwise movement. This movement around the polygon can be performed utilizing nearest neighbor, single well, translations, or by multi- $\Phi_{0}$ traversals between distant wells in the potential, depending on the magnitude of the applied pulse. The actual level in $V_{\text {out }}$ versus $I_{\text {in }}$ into which the system settles is determined by three factors: (i) the well in which the SQUID ring is currently localized, (ii) the size of voltage pulse used to change location in the potential, and (iii) the direction of traversal of the polygon (whether $A$ or $B$-type pulses are utilized).

\section{CONCLUSIONS}

In this work we have demonstrated that, in principle, it is possible to use highly hysteretic (large $\beta$ ) SQUID ring-tank circuit systems as the basis for multilevel logic or memory devices [20-24]. Following earlier experiments, where giant
SQUID magnetometer plateaux were observed containing sets of constant voltage $\left(V_{\text {out }}\right)$ steps, we have shown that the jumping processes between steps on a particular plateau can be generated by either noise on the bias flux $\Phi_{x}$ or voltage pulses applied to the system. For sufficiently low levels of noise we find that a high $\beta$ SQUID ring-tank circuit system can remain stably on one of the steps on any particular plateau until a suitably shaped voltage pulse is applied to the ring. Following such a pulse, the ring can be made to jump in a controlled manner to other steps at different levels of $V_{\text {out }}$. This new level can be either above or below the original voltage level, depending on the present state of the system and the type of pulse used. Provided it is possible to reduce the ambient flux noise on the SQUID ring sufficiently it should prove possible to develop devices with a controlled voltage response which can be selected, and modified, at will. This could prove useful in such areas as multilevel logic or finite state machinery [20-24]. As part of any such developments we would expect to see interesting new fields open up in the nonlinear dynamics of these highly hysteretic systems.

\section{ACKNOWLEDGMENTS}

We would like to thank the Engineering and Physical Sciences Research Council for its generous support of this work. We would also to thank Dr. R.J. Prance and Professor A.R. Bulsara for interesting and informative discussions.
[1] R. J. Prance, R. Whiteman, T. D. Clark, H. Prance, V. Schollmann, J. F. Ralph, S. Al-Khawaja, and M. Everitt, Phys. Rev. Lett. 82, 5401 (1999).

[2] J. R. Friedman, V. Patel, W. Chen, S. K. Tolpygo, and J. E. Lukens, Nature (London) 406, 43 (2000).

[3] C. H. van der Wal, A. C. J. ter Haar, F. K. Wilhelm, R. N. Schouten, C. J. P. M. Harmans, T. P. Orlando, S. Lloyd, and J. E. Mooij, Science 290, 773 (2000).

[4] T. P. Spiller, Fortschr. Phys. 48, 1075 (2000).

[5] I. Chiorescu, Y. Nakamura, C. J. P. M. Harmans, and J. E. Mooij, Science 299, 1869 (2003).

[6] W. C. Schieve, A. R. Bulsara, and E. W. Jacobs, Phys. Rev. A 37, 3541 (1988).

[7] M. P. Soerensen, M. Bartuccelli, P. L. Christiansen, and A. R. Bishop, Phys. Lett. 109A, 347 (1985).

[8] K. Likharev, Dynamics of Josephson Junctions and Circuits (Taylor \& Francis, London, 1986).

[9] L. Chua, C. Desoer, and E. Kuh, Linear and Non-Linear Circuits (McGraw-Hill, New York, 1987).

[10] A. Barone and G. Paterno, Physics and Applications of the Josephson Effect (Wiley, New York, 1982).

[11] T. D. Clark, J. F. Ralph, R. J. Prance, H. Prance, J. Diggins, and R. Whiteman, Phys. Rev. E 57, 4035 (1998).

[12] O. Lounasmaa, Experimental Principles and Methods below $1 K$ (Academic, London, 1974).

[13] J. Zimmerman, P. Thiene, and J. Harding, J. Appl. Phys. 41, 1572 (1970).
[14] L. Gammaitoni, P. Hanggi, P. Jung, and F. Marchesoni, Rev. Mod. Phys. 70, 223 (1998).

[15] A. R. Bulsara and L. Gammaitoni, Phys. Today 49, 39 (1996).

[16] J. F. Lindner, J. Mason, J. Neff, B. J. Breen, W. L. Ditto, and A. R. Bulsara, Phys. Rev. E 63, 041107 (2001).

[17] B. Oksendal, Stochastic Differential Equations: An Introduction with Applications (Springer-Verlag, Berlin and Heidelberg, 2003).

[18] The Langevin Equation with Applications in Physics, Chemistry and Electrical Engineering, edited by W. Coffey, Y. Kalmykov, and J. Waldron, Contemporary Chemical Physics Vol. 10 (World Scientific, Singapore, 1997).

[19] E. Benjacob and D. Abraham, Appl. Phys. Lett. 39, 835 (1981).

[20] R. K. Brayton, G. D. Hachtel, and A. L. Sangiovannivincentelli, Proc. IEEE 78, 264 (1990).

[21] S. Devadas, H. K. Ma, A. R. Newton, and A. Sangiovannivincentelli, IEEE Trans. Comput.-Aided Des. 7, 1290 (1988).

[22] K. Bartlett, W. Cohen, A. Degeus, and G. Hachtel, IEEE Trans. Comput.-Aided Des. 5, 582 (1986).

[23] B. Lin and S. Devadas, IEEE Trans. Comput.-Aided Des. 14, 974 (1995).

[24] R. G. Li, J. N. Wang, Y. Q. Wang, W. F. Dong, and D. X. Wu, Quantum resonant tunneling effect and multi-value logic memory 5th International Conference On Solid-State And Integrated Circuit Technology Proceedings, edited by M. Zhang and K. N. Tu (1998), pp. 588-589. 